\title{
ON GORENSTEIN REES ALGEBRAS OF CODIMENSION THREE
}

\section{YUJI KAMOI}

School of Commerce

Meiji University

1-9-1 Eihuku, Suginami-ku

Tokyo, $168-8555$

Japan

e-mail: kamoi@meiji.ac.jp

\begin{abstract}
The aim of this paper is to determine a structure of an ideal such that its Rees algebra is a Gorenstein ring of codimension three. In order to prove our results, we study a structure of free resolutions of certain grade 2 ideals having projective dimension 2 .
\end{abstract}

\section{Introduction}

Let $(A, \mathfrak{m})$ be a $d$-dimensional Noetherian local ring and $I$ be an ideal of $A$ generated by $a_{1}, \cdots, a_{m}$. We consider a Rees algebra $R(I)=\bigoplus_{n \geq 0} I^{n} t^{n}$ of $I$ and its presentation. Let $S=A\left[T_{1}, \cdots, T_{m}\right]$ be a polynomial ring over $A$ and define an $A$-algebra map $\varphi: S \rightarrow R(I)$ by $\varphi\left(T_{i}\right)=a_{i} t$ for $1 \leq i \leq m$. In this paper, we determine a structure of $I$ such that $R(I)$ is a Gorenstein ring of $\operatorname{pd}_{S}(R(I))=3$. By Buchsbaum2010 Mathematics Subject Classification: 13A30, 13D02, $13 \mathrm{H} 10$.

Keywords and phrases: Gorenstein rings, Rees algebra, Gorenstein ideal of grade 3.

Submitted by Kazufumi Eto.

Received October 8, 2016; Revised November 28, 2016

(C) 2016 Scientific Advances Publishers 
Eisunbud [3], such $R(I)$ is determined by a skew-symmetric matrix and the defining ideal of $R(I)$ is given by pfaffians of this (sub)matrix. Then we characterize such an ideal $I$ by the form of generators of $I$, using entries of the presentation matrix of $R(I)$.

To describe our result, we give some notations. Let $\mathbf{x}=\left(x_{i}\right)_{1 \leq i \leq 4}$ be a $1 \times 4 A$-matrix and $Y=\left(y_{i j}\right)_{1 \leq i \leq 4,1 \leq j \leq 6}$ be a $4 \times 6 A$-matrix. For $1 \leq i<$ $j<k \leq 4$ and $1 \leq p<q<r \leq 6$, we denote the 3-minor of $Y$ by $[i j k ; p q r]=\left|\begin{array}{ccc}y_{i p} & y_{i q} & y_{i r} \\ y_{j p} & y_{j q} & y_{j r} \\ y_{k p} & y_{k q} & y_{k r}\end{array}\right|$. For $1 \leq i<j<k \leq 4$, we put a quadratic form of $\mathbf{x}$

$$
Q(i j k)=\mathbf{x} \cdot\left(\begin{array}{cccc}
{[i j k ; 123]} & {[i j k ; 125]} & {[i j k ; 126]} & {[i j k ; 136]} \\
-[i j k ; 134] & {[i j k ; 145]} & {[i j k ; 146]} & {[i j k ; 156]} \\
-[i j k ; 234] & {[i j k ; 245]} & {[i j k ; 246]} & {[i j k ; 256]} \\
-[i j k ; 235] & {[i j k ; 345]} & {[i j k ; 346]} & {[i j k ; 356]}
\end{array}\right) \cdot \mathbf{x}^{t},
$$

and denote by $I(\mathbf{x} ; Y)=(Q(123), Q(124), Q(134), Q(234))$. Then our main result state as follows.

Theorem 1.1. Suppose that $A$ is a Gorenstein ring.

(1) If (i) $I(\mathbf{x} ; Y)$ is generically a complete intersection of $\operatorname{ht}(I(\mathbf{x} ; Y))=2$, (ii) $r\left(I(\mathbf{x} ; Y)_{\mathfrak{p}}\right) \leq 1$ for $\mathfrak{p} \in V(I(\mathbf{x} ; Y))$, ht $(\mathfrak{p})=3$, and (iii) $\operatorname{ht}((\mathbf{x})) \geq 3$, then $R(I(\mathbf{x} ; Y))$ is a Gorenstein ring of $\operatorname{pd}_{S}(R(I(\mathbf{x} ; Y)))=3$.

(2) Conversely, for generically a complete intersection ideal I of $r(I)>0$, if $R(I)$ is a Gorenstein ring of $\operatorname{pd}_{S}(R(I))=3$, then there exist a $1 \times 4$ matrix $\mathbf{x}$ and $a 4 \times 6$ matrix $Y$ satisfying conditions that (i) $I=I(\mathbf{x} ; Y)$ and $\operatorname{ht}(I)=2$, (ii) $r\left(I_{\mathfrak{p}}\right) \leq 1$ for $\mathfrak{p} \in V(I)$, ht $(\mathfrak{p})=3$, and (iii) $\operatorname{ht}((\mathbf{x})) \geq 3$. 
Here we denote by $r(I)$ a reduction number of $I$.

\section{Motivations and Preliminary}

According to many study of Rees algebras, it seems that the Gorenstein property of $R(I)$ gives very strong restriction for $I$. For example, if $I$ is generically a complete intersection ideal of $h t(I) \geq 2$, then the Gorenstein property of $R(I)$ implies that $\mathrm{ht}(I)=2$. Moreover, if $I$ have a relatively small analytic deviation and $R(I)$ is Gorenstein, then $I$ is characterized by a explicit conditions (cf. [7], [5], [4], et al.).

Then we expect to give a structure of generically complete intersection ideals of height two such that $R(I)$ is Gorenstein. At this point of view, the first case we have to take into consideration is a complete intersection (CI) property of $R(I)$ and, in fact, this property of $R(I)$ gives very clear description of $I$ by results of Avramov [1], Huneke [8], [9], and Simis et al. [13], as below.

Theorem 2.1 (Proposition 9 [1], Theorem 3.1 [8], Theorem 2.3 [9], Theorem 3.8 [13]).

Suppose that $A$ is a Gorenstein ring and $\mathrm{ht}(I) \geq 2$. Then the following conditions are equivalent:

(1) $R(I)$ is $r C I$ (i.e., $\operatorname{Ker}(\varphi)$ is generated by a regular sequence).

(2) $R(I)$ is a Gorenstein ring and $I$ is generically a complete intersection perfect ideal.

(3) $R(I)$ is a Gorenstein ring and I is a perfect ideal of $\mathrm{ht}(I)=2$.

(4) $G(I)$ is a Cohen-Macaulay ring with $a(G(I))=-\mathrm{ht}(I)$ and $I$ is a perfect ideal of $\mathrm{ht}(I)=2$.

(5) I is a perfect ideal of $\mathrm{ht}(I)=2$ such that $\mu\left(I_{\mathfrak{p}}\right) \leq \mathrm{ht}(\mathfrak{p})$ for all $\mathfrak{p} \in V(I)$. 
(6) I is a perfect ideal of $\mathrm{ht}(I)=2$ and is generated by a d-sequence.

(7) $I=I_{m-1}(X)$ for some $m-1 \times m$ A-matrix $X$ such that grade $\left(I_{t}(X)\right) \geq m+1-t$ for $1 \leq t \leq m$.

As a next step, several author examine to study an interaction between homological properties of $R(I)$ and a defining equation, using a Jacobian dual of a presentation of I (Morey [11], Morey and Ulrich [12], Ulrich and Vasconcelos [14]). Our main result gives a simple and a concrete application of their approach, (indirectly) and it completely generalize Theorem 8.2.18 of Vasconcelos [15].

In the rest of this section, we give some notations and basic results. Let $R$ be a commutative ring and let $\phi: F \rightarrow G$ be an $R$-linear map of free modules. If we fix free basis $\mathbf{f}_{1}, \cdots, \mathbf{f}_{m} \in F, \mathbf{g}_{1}, \cdots, \mathbf{g}_{n} \in G$ and $\phi\left(\mathbf{f}_{i}\right)=\sum_{j=1}^{n} a_{i j} \mathbf{g}_{j}$ for all $1 \leq i \leq m$, then we identify $\phi$ with an $m \times n \quad R$-matrix $\left(a_{i j}\right)_{\substack{1 \leq i \leq m \\ 1 \leq j \leq n}}$. Note that a matrix of a composite map $\psi \circ \phi: F \stackrel{\phi}{\rightarrow} G \stackrel{\psi}{\rightarrow} H$ of free modules corresponds to $\phi \cdot \psi$.

Let $\mathbf{x}=x_{1}, \cdots, x_{n} \in R$ be a sequence of $R$. We denote a Koszul complex w.r.t. $\mathbf{x}$ by $\left(K_{\bullet}(\mathbf{x}), \partial_{\bullet}\right)$ and $K_{1}(\mathbf{x})=\bigoplus_{i=1}^{n} \operatorname{Re}_{i}$. We put $Z_{i}(\mathbf{x})=\operatorname{Ker}\left(\partial_{i}\right)$ and $B_{i}(\mathbf{x})=\operatorname{Im}\left(\partial_{i+1}\right)$ for $i=0, \cdots, n$. For an $R$-linear $\bigoplus_{i=1}^{n} \operatorname{Re}_{i} \stackrel{\phi}{\rightarrow} R$, we also denote by $K_{\bullet}(\phi)=K_{\bullet}\left(\phi\left(\mathbf{e}_{1}\right), \cdots, \phi\left(\mathbf{e}_{n}\right)\right)\left(\right.$ resp., $Z_{\bullet}(\phi)=Z_{\bullet}\left(\phi\left(\mathbf{e}_{1}\right), \cdots\right.$, $\left.\left.\phi\left(\mathbf{e}_{n}\right)\right), B_{\bullet}(\phi)=B_{\bullet}\left(\phi\left(\mathbf{e}_{1}\right), \cdots, \phi\left(\mathbf{e}_{n}\right)\right)\right)$. Recall that an element $\alpha \in B_{1}(\mathbf{x})$ is represented by a skew-symmetric matrix $N$ such that $\alpha=\mathbf{x} \cdot N$. Moreover, if $n=4$, then we have the following description of $\alpha$.

Lemma 2.2 (cf. Example 2.7 [10]). Suppose that $n=4$ and $\alpha=\sum_{i=1}^{4} a_{i} \mathbf{e}_{i} \in K_{1}(\mathbf{x})$. Then the following are equivalent:

(1) $\alpha$ is an element of $B_{1}(\mathbf{x})$ and $\alpha=\sum_{i<j} c_{i j} \partial_{2}\left(\mathbf{e}_{i} \wedge \mathbf{e}_{j}\right)$. 
(2) For $1 \leq i \leq 4, a_{i}=(-1)^{i} \mathrm{pf}_{i+1}(M)$, where

$$
M=\left(\begin{array}{c|cccc}
0 & x_{1} & x_{2} & x_{3} & x_{4} \\
\hline-x_{1} & 0 & c_{34} & -c_{24} & c_{23} \\
-x_{2} & -c_{34} & 0 & c_{14} & -c_{13} \\
-x_{3} & c_{24} & -c_{14} & 0 & c_{12} \\
-x_{4} & -c_{23} & c_{13} & -c_{12} & 0
\end{array}\right) .
$$

Proof. By the identity pf $\left(\begin{array}{cccc}0 & a & b & c \\ -a & 0 & d & e \\ -b & -d & 0 & f \\ -c & -e & -f & 0\end{array}\right)=a f-b e+c d$, it is easy to compute $\mathrm{pf}_{\bullet}(M)$ and we have that

$$
\begin{aligned}
\left(\begin{array}{r}
-\mathrm{pf}_{2}(M) \\
\mathrm{pf}_{3}(M) \\
-\mathrm{pf}_{4}(M) \\
\mathrm{pf}_{5}(M)
\end{array}\right) & \left(\begin{array}{ccc}
-c_{12} x_{2} & -c_{13} x_{3} & -c_{14} x_{4} \\
c_{12} x_{1} & -c_{23} x_{3} & -c_{24} x_{4} \\
c_{13} x_{1} & +c_{23} x_{2} & -c_{34} x_{4} \\
c_{14} x_{1} & +c_{24} x_{2} & +c_{34} x_{3}
\end{array}\right) \\
& =\left(\begin{array}{cccc}
0 & -c_{12} & -c_{13} & -c_{14} \\
c_{12} & 0 & -c_{23} & -c_{24} \\
c_{13} & c_{23} & 0 & -c_{34} \\
c_{14} & c_{24} & c_{34} & 0
\end{array}\right)\left(\begin{array}{l}
x_{1} \\
x_{2} \\
x_{3} \\
x_{4}
\end{array}\right)\left(\begin{array}{l}
a_{1} \\
a_{2} \\
a_{3} \\
a_{4}
\end{array}\right)=\alpha^{t} .
\end{aligned}
$$


Lemma 2.3. Let $\psi: R \rightarrow T$ be a surjective homomorphism of Noetherian graded rings such that $R_{0}=T_{0}=A$. For any prime ideal $\mathfrak{p} \subset A$ and any integer $n$, if either $\operatorname{depth}_{A_{\mathfrak{p}}}\left(\left[R_{n}\right]_{\mathfrak{p}}\right)>0$ or $\left[\operatorname{Ker}\left(\psi_{\mathfrak{p}}\right)\right]_{n}=(0)$, then $\psi$ is an isomorphism.

Proof. If $\psi$ is not an isomorphism, then there exists an integer $n>0$ such that $[\operatorname{Ker}(\psi)]_{n} \neq(0)$. Then, for $\mathfrak{p} \in A s s_{A}\left(\operatorname{Ker}(\psi)_{n}\right)$, we have that $\left[\operatorname{Ker}\left(\psi_{\mathfrak{p}}\right)\right]_{n} \neq(0)$ and $\operatorname{depth}_{A_{\mathfrak{p}}}\left(\left[R_{\mathfrak{p}}\right]_{n}\right)=0$.

Lemma 2.4. Let $I \subset R$ be an ideal of $\operatorname{grade}(I)=2$. Suppose that $R / I$ has a following $R$-free resolution (not necessarily minimal): $0 \rightarrow R \stackrel{\phi_{3}}{\rightarrow} R^{n} \stackrel{\phi_{2}}{\rightarrow} R^{n} \stackrel{\phi_{1}}{\rightarrow} R$ such that $\operatorname{Im}\left(\phi_{2}^{t}\right) \subset B_{1}\left(\phi_{3}^{t}\right)$. For a prime ideal $\mathfrak{p}$, if $\mu\left(I_{\mathfrak{p}}\right)<n$, then $I_{\mathfrak{p}}$ is perfect.

Proof. Since $\operatorname{Im}\left(\phi_{2}^{t}\right) \subset B_{1}\left(\phi_{3}^{t}\right) \subset I_{1}\left(\phi_{3}\right) K_{1}\left(\phi_{3}^{t}\right)$, we have $I_{1}\left(\phi_{2}\right) \subset I_{1}\left(\phi_{3}\right)$. It follows that $\left(\phi_{3}\right)_{\mathfrak{p}}$ splits, if $\mu\left(I_{\mathfrak{p}}\right)<n$.

\section{Relations of Rees Algebras}

Throughout this section, we assume that $(A, \mathfrak{m})$ is a $d$-dimensional Gorenstein local ring and $I$ is generically a complete intersection ideal of $\operatorname{ht}(I) \geq 2$. Also, we regard $R(I)$ and $S$ as graded rings by $\operatorname{deg}(t)=1$ and $\operatorname{deg}\left(T_{i}\right)=1$ for all $i$. In order to prove Theorem 1.1, we characterize the Gorenstein property of $R(I)$ such that $\operatorname{pd}_{S}(R(I))=3$ in terms of the free resolution of $I$, below.

Theorem 3.1. (1) If $\mathrm{ht}(I)=2, r\left(I_{\mathfrak{p}}\right) \leq 1$ for all $\mathfrak{p} \in V(I)$ of $\mathrm{ht}(\mathfrak{p}) \leq 3$

and $A / I$ has a free resolution of the form $0 \rightarrow A \stackrel{\phi_{3}}{\rightarrow} A^{4} \stackrel{\phi_{2}}{\rightarrow} A^{4} \stackrel{\phi_{1}}{\rightarrow} A$ s.t. $\operatorname{Im}\left(\phi_{2}^{t}\right) \subset B_{1}\left(\phi_{3}^{t}\right)$, then $R(I)$ is a Gorenstein ring of $\operatorname{pd}_{S}(R(I))=3$. 
(2) Conversely, if $r(I)>0$ and $R(I)$ is a Gorenstein ring of $\operatorname{pd}_{S}(R(I))=3$, then $\operatorname{ht}(I)=2, r\left(I_{\mathfrak{p}}\right) \leq 1$ for all $\mathfrak{p} \in V(I)$ of $\operatorname{ht}(\mathfrak{p}) \leq 3$ and $A$ / I has a free resolution of the form $0 \rightarrow A \stackrel{\phi_{3}}{\rightarrow} A^{4} \stackrel{\phi_{2}}{\rightarrow} A^{4} \stackrel{\phi_{1}}{\rightarrow} A$ such that $\operatorname{Im}\left(\phi_{2}^{t}\right) \subset B_{1}\left(\phi_{3}^{t}\right)$.

Corollary 3.2 (cf. Theorem 8.2.18, [15]). Suppose that $I_{\mathfrak{p}}$ is unmixed for all $\mathfrak{p} \in V(I)$ of $\mathrm{ht}(\mathfrak{p})=3$. Then the following conditions are equivalent:

(1) $r(I)>0$ and $R(I)$ is a Gorenstein ring of $\operatorname{pd}_{S}(R(I))=3$.

(2) $r(I)=1, \operatorname{ht}(I)=2, \mu\left(I_{\mathfrak{p}}\right) \leq \mathrm{ht}(\mathfrak{p})$ for all $\mathfrak{p} \in V(I)$ of $\mathrm{ht}(\mathfrak{p}) \leq 3$ and $A$ / I has a free resolution of the form $0 \rightarrow A \stackrel{\phi_{3}}{\rightarrow} A^{4} \stackrel{\phi_{2}}{\rightarrow} A^{4} \stackrel{\phi_{1}}{\rightarrow} A$.

Proof of Theorem 3.1, (2). In general, if $R(I)$ is a Cohen-Macaulay ring of $\operatorname{pd}_{S}(R(I))<\infty$, then $\operatorname{pd}_{S}(R(I))=\mu(I)-1$, since $\operatorname{dim}(R(I))=d+1$. In particular, if $R(I)$ is a Gorenstein ring of $\operatorname{pd}_{S}(R(I))=3$, then $\mu(I)=\operatorname{pd}_{S}(R(I))+1=4$.

Let $\mathbb{F}_{\bullet}: 0 \rightarrow F_{m-1} \rightarrow \cdots \rightarrow F_{1} \rightarrow F_{0}=S$ be a grade minimal $S$-free resolution of $R(I)$ and denote the number of the maximal degree of homogeneous basis of $F_{i}$ by $d_{i}=\max \left\{n \mid\left[F_{i} / S_{+} F_{i}\right]_{n} \neq 0\right\}$. Since $S(-m)$ - dual $\mathbb{F}_{\bullet}^{*}$ of $\mathbb{F}_{\bullet}$ gives a graded minimal free resolution of $K_{R(I)}$, the number of the minimal degree of homogeneous basis of $F_{i}^{*}$ is $m-d_{i}$. By the minimality of the resolution, we have $m-d_{i-1} \geq m-d_{i}$ for $1 \leq i<m$ and, thus, $d_{i-1} \leq d_{i}$ for $1 \leq i<m$. Also, since $a(R(I))=-1$, if $R(I)$ is a Gorenstein, then we have $d_{\mu(I)-1}=\mu(I)+a(R(I))=\mu(I)-1 \geq$ $d_{i}$ for all $i$. This conclude that if $R(I)$ is a Gorenstein of $\operatorname{pd}_{S}(R(I))=3$, then there is a minimal $S$-free resolution of the form 


$$
\mathbb{F}_{\bullet}=0 \rightarrow S(-3) \stackrel{\Psi_{3}}{\rightarrow} S^{b+3}(-2) \oplus S^{b}(-1) \stackrel{\Psi_{2}}{\rightarrow} S^{b}(-2) \oplus S^{b+3}(-1) \stackrel{\Psi_{1}}{\rightarrow} S
$$

for some $b \geq 0$, since $\left[\mathbb{F}_{\bullet}\right]_{1}$ induces a minimal $A$-free resolution of $I$;

$$
\left[\mathbb{F}_{\bullet}\right]_{1}=0 \rightarrow A^{b} \stackrel{\phi_{3}}{\rightarrow} A^{b+3} \stackrel{\phi_{2}}{\rightarrow} A^{4}
$$

Then, by [3], we may choose $\Psi_{\bullet}$ as $\Psi_{2}=\left(\begin{array}{c|c}0 & \phi_{3} \\ \hline-\phi_{3}^{t} & \Phi\end{array}\right)$ and

$$
\Psi_{1}^{t}=\Psi_{3}=\left(\operatorname{pf}_{1}\left(\Psi_{2}\right) \quad \operatorname{pf}_{2}\left(\Psi_{2}\right) \cdots \operatorname{pf}_{b}\left(\Psi_{2}\right) \quad\left(T_{1} \cdots T_{4}\right) \phi_{2}^{t}\right),
$$

where $\Phi: S^{b+3}(-2) \rightarrow S(-1)^{b+3}$ is alternating.

Now, suppose that $b \geq 2$. Since $I_{1}\left(\phi_{3}\right) \subset \mathfrak{m}$, we have $\operatorname{pf}_{i}\left(\Psi_{2}\right)^{2} \in \mathfrak{m} S$ for $1 \leq i \leq b$, namely, $\operatorname{pf}_{i}\left(\Psi_{2}\right) \in \mathfrak{m} S$ for $1 \leq i \leq b$. Hence $\operatorname{Ker}(\varphi) \subset \mathfrak{m} S$. On the other hand, for some minimal reduction $J \subset I$ with $r=r_{J}(I)>0$, $I^{r+1}=J I^{r}$ implies $[\operatorname{Ker}(\varphi)]_{r+1} \not \subset[\mathfrak{m} S]_{r+1}$. This is a contradiction and $b=1$. Thus, we obtain a minimal free resolution of $A / I$ as

$$
0 \rightarrow A \stackrel{\phi_{3}}{\rightarrow} A^{4} \stackrel{\phi_{2}}{\rightarrow} A^{4} \stackrel{\phi_{1}}{\rightarrow} A
$$

By Lemma 2.2, if we put $f_{i}=(-1)^{i} \operatorname{pf}_{i+1}\left(\Psi_{2}\right)$ for $i=1,2,3,4$, then we have that $\left(f_{1}, f_{2}, f_{3}, f_{4}\right)=\left(T_{1} T_{2} T_{3} T_{4}\right) \cdot \phi_{2}^{t} \in B_{1}\left(\phi_{3}^{t} ; S\right)=B_{1}\left(\phi_{3}^{t}\right) \otimes_{A} S$. This induces that $\operatorname{Im}\left(\phi_{2}^{t}\right) \subset B_{1}\left(\phi_{3}^{t} ; A\right)$.

Finally, since $I$ is generically a complete intersection, $\operatorname{ht}(I)=2$ and $r\left(I_{\mathfrak{p}}\right)=0$ for $\mathfrak{p} \in \operatorname{Min}(A / I)$. Also, by (1.1)[5], $r\left(I_{\mathfrak{p}}\right) \leq 1$ for all $\mathfrak{p} \in V(I)$ of $h t(\mathfrak{p})=3$. This complete the proof of Theorem 3.1, (2). 
Proof of Theorem 3.1, (1). Let $0 \rightarrow A \stackrel{\phi_{3}}{\rightarrow} A^{4} \stackrel{\phi_{2}}{\rightarrow} A^{4} \stackrel{\phi_{1}}{\rightarrow} A$ be a free resolution of $A / I$ such that $\operatorname{Im}\left(\phi_{2}^{t}\right) \subset B_{1}\left(\phi_{3}^{t}\right)$. If we denote by $\phi_{3}=\left(\begin{array}{llll}x_{1} & x_{2} & x_{3} & x_{4}\end{array}\right) \quad$ and $\quad \mathbf{f}^{t}=\left(\begin{array}{llll}f_{1} & f_{2} & f_{3} & f_{4}\end{array}\right)=\left(\begin{array}{llll}T_{1} & T_{2} & T_{3} & T_{4}\end{array}\right) \cdot \phi_{2}^{t}$, then $\mathbf{f}^{t} \in B_{1}\left(\phi_{3}^{t} ; S\right)=B_{1}\left(\phi_{3}^{t}\right) \otimes_{A} S$ by our assumption. Then, by Lemma 2.2, we have a $5 \times 5 S$-matrix

$M=\left(\begin{array}{c|cccc}0 & x_{1} & x_{2} & x_{3} & x_{4} \\ \hline-x_{1} & 0 & c_{34} & -c_{24} & c_{23} \\ -x_{2} & -c_{34} & 0 & c_{14} & -c_{13} \\ -x_{3} & c_{24} & -c_{14} & 0 & c_{12} \\ -x_{4} & -c_{23} & c_{13} & -c_{12} & 0\end{array}\right)$,

such that $\left(f_{1} f_{2} f_{3} f_{4}\right)=\left(-\operatorname{pf}_{2}(M) \quad \mathrm{pf}_{3}(M)-\mathrm{pf}_{4}(M) \quad \mathrm{pf}_{5}(M)\right)$. If we put $g=\mathrm{pf}_{1}(M)$ and consider the relation of pfaffians, then $-x_{i} g \in\left(f_{1}, f_{2}\right.$, $\left.f_{3}, f_{4}\right) \subset \operatorname{Ker}(\varphi)$. Since $\left(x_{1}, \cdots, x_{4}\right)$ contains a nonzero divisor of $R(I) \subset A[t]$, we have $g \in \operatorname{Ker}(\varphi)$ and $\mathfrak{a}:=\operatorname{Pf}(M) \subset \operatorname{Ker}(\varphi)$. On the other hand, a Krull dimension of $S /\left(f_{1}, f_{2}, f_{3}, f_{4}\right) \cong \operatorname{Sym}(I)$ is $\sup _{\mathfrak{p}}\left\{\operatorname{dim}(A / \mathfrak{p})+\mu\left(I_{\mathfrak{p}}\right)\right\}=d+1$ and $3=\operatorname{ht}\left(f_{1}, f_{2}, f_{3}, f_{4}\right) \leq \operatorname{ht}(\mathfrak{a})$. Since $\operatorname{dim}(S / \mathfrak{a}) \geq \operatorname{dim}(R(I))=d+1, S / \mathfrak{a}$ is a Gorenstein ring of $\operatorname{pd}_{S}(S / \mathfrak{a})=3$.

It is easy to show that $(S / \mathfrak{a})_{\mathfrak{p}} \cong R(I)_{\mathfrak{p}}$ for all $\mathfrak{p} \notin V(I)$, since $\left(f_{1}, f_{2}\right.$, $\left.f_{3}, f_{4}\right) \subset \mathfrak{a}$. Thus, in order to prove $S / \mathfrak{a} \cong R(I)$, it is enough to show $\operatorname{depth}\left(\left[S_{\mathfrak{p}} / \mathfrak{a}_{\mathfrak{p}}\right]_{n}\right)>0$ for all $\mathfrak{p} \in V(I)$ and all $n>0$, by Lemma 2.3. Since $I$ is generically a complete intersection, $R\left(I_{\mathfrak{p}}\right) \cong S_{\mathfrak{p}} / \mathfrak{a}_{\mathfrak{p}}$ for $\operatorname{ht}(\mathfrak{p})=2$. Also, we have that $\operatorname{pd}_{A}\left([S / \mathfrak{a}]_{n}\right) \leq 3$ for all $n>0$. It follows that the assertion is trivial for $\operatorname{ht}(\mathfrak{p}) \neq 3$. Then it is enough to show that $\operatorname{depth}_{A}\left(S_{\mathfrak{p}} / \mathfrak{a}_{\mathfrak{p}}\right)>0$ for $\operatorname{ht}(\mathfrak{p})=3$ and, through the localization at $\mathfrak{p}$, we 
may assume that $\operatorname{dim}(A)=3$. Note that $r(I) \leq 1$ by our assumption. If $r(I)=0$, then $\mu(I) \leq 3$ and by Lemma 2.4, $I$ is Cohen-Macaulay. Hence $R(I) \cong \operatorname{Sym}(I) \cong S / \mathfrak{a}$ and $\operatorname{depth}_{A}(S / \mathfrak{a})>0$.

Finally, we may assume that $r(I)=1$ and let

$$
\mathbb{F}=0 \rightarrow S(-3) \stackrel{\Phi_{3}}{\rightarrow} S^{4}(-2) \oplus S(-1) \stackrel{\Phi_{2}}{\rightarrow} S(-2) \oplus S(-1)^{4} \stackrel{\Phi_{1}}{\rightarrow} S
$$

be a graded $S$-free resolution of $S / \mathfrak{a}$. Then we have an exact sequence

$$
0 \rightarrow \operatorname{Hom}_{A}(A / \mathfrak{m}, S / \mathfrak{a}) \rightarrow \operatorname{Ext}_{A}^{3}(A / \mathfrak{m}, S)(-3) \stackrel{\Phi_{3}^{\prime}}{\rightarrow} \operatorname{Ext}_{A}^{3}(A / \mathfrak{m}, S)(-1)
$$

$$
\operatorname{Ext}_{A}^{3}\left(A / \mathfrak{m}, S^{4}\right)(-2)
$$

since $\operatorname{Hom}_{A}(A / \mathfrak{m}, S / \mathfrak{a}) \cong \operatorname{Ext}_{A}^{2}\left(A / \mathfrak{m}, \operatorname{Im}\left(\Phi_{2}\right)\right)$. It follows that $[S / \mathfrak{a}]_{2} \cong I^{2}$, since $\left[\operatorname{Hom}_{A}(A / \mathfrak{m}, S / \mathfrak{a})_{2}=0\right.$. Also we have that $S_{2} / \mathfrak{m} S_{2} \nRightarrow I^{2} / \mathfrak{m} I^{2}$, since $r(I)=1$ and, thus $g \notin \mathfrak{m} S$. Now, $\Phi_{3}^{\prime}$ is a multiplication of $g$, by the form of the matrix $\Phi_{3}$. This conclude that $\Phi_{3}^{\prime}$ is injective and $\operatorname{Hom}_{A}(A / \mathfrak{m}, S / \mathfrak{a})=(0)$.

Proof of Corollary 3.2. (1) $\Rightarrow(2)$. For $\mathfrak{p} \in V(I)$ such that $h t(\mathfrak{p})=3$, $A_{\mathfrak{p}} / I_{\mathfrak{p}}$ is Cohen-Macaulay, since $I$ is generically a complete intersection of $\operatorname{ht}(I)=2$ and $I_{\mathfrak{p}}$ is unmixed. Then by Theorem $2.1, \mu\left(I_{\mathfrak{p}}\right) \leq \operatorname{ht}(\mathfrak{p})$ for all $\mathfrak{p} \in V(I)$ of $h t(\mathfrak{p}) \leq 3$. Also, by the proof of Theorem 3.1, (2), $R(I)$ is defines three linear forms and one quadratic form. (See Lemma 2.2.) Namely, the fiber cone $F(I)$ is hypersurface defined by the degree 2 polynomial. Hence, we have $r(I)=a(F(I))+\ell(I)=2-4+3=1$ and the assertion follows from Theorem 3.1, (2). 
$(2) \Rightarrow(1)$. For $\mathfrak{p} \in V(I)$ of $\mathrm{ht}(\mathfrak{p}) \leq 3, I_{\mathfrak{p}}$ is perfect by our assumption. Then $\mu\left(I_{\mathfrak{p}}\right) \leq \operatorname{ht}(\mathfrak{p})$ implies $r\left(I_{\mathfrak{p}}\right)=0$ by Theorem 2.1. Furthermore, $\left(\phi_{3}\right)_{\mathfrak{p}}$ splits for all $\mathfrak{p} \in V(I)$ of $\mathrm{ht}(\mathfrak{p}) \leq 3$. It follows that grade $\left(I_{1}\left(\phi_{3}\right)\right)=4$ and $\operatorname{Im}\left(\phi_{2}^{t}\right) \subset Z_{1}\left(\phi_{3}^{t}\right)=B_{1}\left(\phi_{3}^{t}\right)$.

Remark 3.3. As in the proof of Theorem 3.1, (1); Theorem 1.1, (ii) can be replaced by conditions that $h t\left(I_{1}\left(\phi_{3}\right)+c(g)\right) \geq 4$ and $r\left(I_{\mathfrak{p}}\right)=0$ for all $\mathfrak{p} \in V(I) \backslash V\left(I_{1}\left(\phi_{3}\right)\right)$ of $\operatorname{ht}(\mathfrak{p})=3$. Here $c(g)$ is a content ideal of $g$. Note that, in Theorem 1.1, (2), $r(I)>0$ implies $c(g)=A$ as above.

\section{Ideals of Betti Type $(1, n, n, 1)$}

Let $R$ be a Noetherian ring. In this section, we study ideals having free resolutions of the form $0 \rightarrow R \rightarrow R^{n} \rightarrow R^{n} \rightarrow R$. In Theorem 4.1, we reconstruct these free resolutions from Koszul relations. Furthermore, in Theorem 4.6, we give a structure of a certain ideals having these free resolutions. First note that, by [2], an $R$-free complex $0 \rightarrow R \stackrel{\phi_{3}}{\rightarrow} R^{n} \stackrel{\phi_{2}}{\rightarrow} R^{n} \stackrel{\phi_{1}}{\rightarrow} R$ is acyclic if and only if (i) $\operatorname{grade}\left(I_{1}\left(\phi_{1}\right)\right)>0$, (ii) $\operatorname{rk}\left(\phi_{2}\right)=n-1$ and $\operatorname{grade}\left(I_{n-1}\left(\phi_{2}\right)\right) \geq 2$, and (iii) $\operatorname{grade}\left(I_{1}\left(\phi_{3}\right)\right) \geq 3$.

Now, we consider the Koszul relation of $\phi_{3}$. Let $\mathbf{x}=x_{1}, \cdots, x_{n} \in R$ be a sequence of elements and let $\left(K_{\bullet}(\mathbf{x}), \partial_{\bullet}\right)$ be a Koszul complex with respect to $\mathbf{x}$. We denote by $K_{\bullet}(\mathbf{x})^{*}=\operatorname{Hom}_{R}\left(K_{\bullet}(\mathbf{x}), R\right)$. For a free basis $\mathbf{e}_{1}, \cdots, \mathbf{e}_{n}$ of $K_{1}(\mathbf{x})$, we put $\mathbf{e}_{i}^{c}=\bigwedge_{j \neq i} \mathbf{e}_{j} \in K_{n-1}(\mathbf{x})$ for $1 \leq i \leq n$ and define a map $s: K_{1}(\mathbf{x})^{*} \rightarrow K_{n-1}(\mathbf{x})$ by $s\left(\mathbf{e}_{i}^{*}\right)=(-1)^{i-1} \mathbf{e}_{i}^{c}$ for $i=1, \cdots, n$.

Let $\phi: K_{1}(\mathbf{x}) \rightarrow K_{1}(\mathbf{x})$ be an $R$-linear map such that $\partial_{1} \circ \phi=0$. Then, we have $\operatorname{Im}(\stackrel{i}{\wedge} \phi) \subset \operatorname{Ker}\left(\partial_{i}\right)$ for all $i>0$. Thus, if grade $((\mathbf{x}))=k$, 
then there exist $\tau_{i+1}: K_{i}(\mathbf{x}) \rightarrow K_{i+1}(\mathbf{x})$ such that $\stackrel{i}{\wedge} \phi=\partial_{i+1} \circ \tau_{i+1}$ for all $i \geq n-k+1$. In particular, we put $\tau(\mathbf{x} ; \phi)=\tau_{n}: K_{n-1}(\mathbf{x}) \rightarrow K_{n}(\mathbf{x}) \cong R$. Then ideals of the betti type $(1, n, n, 1)$ coincide with $I_{1}(\tau(\mathbf{x} ; \phi))$.

Theorem 4.1. (1) If grade $((\mathbf{x})) \geq 3$ and $\operatorname{grade}\left(I_{1}(\tau(\mathbf{x} ; \phi))\right) \geq 2$, then $a$ sequence

$$
0 \rightarrow K_{0}(\mathbf{x})^{*} \stackrel{\partial_{1}^{*}}{\rightarrow} K_{1}(\mathbf{x})^{*} \stackrel{\phi^{*}}{\rightarrow} K_{1}(\mathbf{x})^{*} \stackrel{\tau(\mathbf{x} ; \phi) \circ s}{\longrightarrow} K_{n}(\mathbf{x})
$$

is an acyclic complex.

(2) Conversely, if $I \subset R$ is an ideal with grade $(I) \geq 2$ such that $R / I$ has a free resolution of the form $0 \rightarrow R \stackrel{\phi_{3}}{\rightarrow} R^{n} \stackrel{\phi_{2}}{\rightarrow} R^{n} \stackrel{\phi_{1}}{\rightarrow} R$ and $\phi_{3}=\left(x_{i}\right)_{1 \leq i \leq n}$, then $I=I_{1}\left(\tau\left(\mathbf{x} ; \phi_{2}^{*}\right)\right)$.

To prove Theorem 4.1, we first show that

$$
0 \rightarrow K_{0}(\mathbf{x})^{*} \stackrel{\partial_{1}^{*}}{\rightarrow} K_{1}(\mathbf{x})^{*} \stackrel{\phi^{*}}{\rightarrow} K_{1}(\mathbf{x})^{*} \stackrel{\tau(\mathbf{x} ; \phi) \circ s}{\longrightarrow} K_{n}(\mathbf{x})
$$

is a complex. This follows from a well-known map of the Laplace expansion of $\operatorname{det}(\phi)$.

Lemma 4.2. (1) An R-linear $\operatorname{map} K_{1}(\mathbf{x})^{*} \stackrel{\phi^{*}}{\rightarrow} K_{1}(\mathbf{x})^{*} \stackrel{s}{\rightarrow} K_{n-1}(\mathbf{x}) \stackrel{n-1}{\longrightarrow}$ $K_{n-1}(\mathbf{x})$ coincides with $K_{1}(\mathbf{x})^{*} \stackrel{\operatorname{det}(\phi) \cdot s}{\longrightarrow} K_{n-1}(\mathbf{x})$.

(2) Dually, an R-linear map $K_{1}(\mathbf{x}) \stackrel{\phi}{\rightarrow} K_{1}(\mathbf{x}) \stackrel{s^{*}}{\rightarrow} K_{n-1}(\mathbf{x})^{*} \stackrel{n-1}{\wedge} \phi^{*} K_{n-1}(\mathbf{x})^{*}$ coincides with $K_{1}(\mathbf{x}) \stackrel{\operatorname{det}(\phi) \cdot s^{*}}{\longrightarrow} K_{n-1}(\mathbf{x})^{*}$.

Proof. (1) We put $\phi=\left(a_{i j}\right)_{1 \leq i, j \leq n}$ and denote by $\phi_{k l}$ an $(n-1)$ square matrix obtained by deleting the $k$-th row and the $l$-th column of $\phi$. Then we have equations 


$$
\begin{aligned}
\left(\begin{array}{c}
n-1 \\
\wedge
\end{array}\right) \circ s \circ \phi^{*}\left(\mathbf{e}_{i}^{*}\right) & =(\stackrel{n-1}{\wedge} \phi) s\left(\sum_{j=1}^{n} a_{j i} \mathbf{e}_{j}^{*}\right)=\wedge \phi\left(\sum_{j=1}^{n}(-1)^{j-1} a_{j i} \mathbf{e}_{j}^{c}\right) \\
& =\sum_{j=1}^{n}(-1)^{j-1} a_{j i} \underset{k \neq j}{\wedge} \phi\left(\mathbf{e}_{k}\right) \\
& =\sum_{j=1}^{n}(-1)^{j-1} a_{j i}\left(\sum_{k=1}^{n} \operatorname{det}\left(\phi_{j k}\right) \mathbf{e}_{k}^{c}\right) \\
& =\sum_{k=1}^{n}\left(\sum_{j=1}^{n}(-1)^{j-1} a_{j i} \operatorname{det}\left(\phi_{j k}\right)\right) \mathbf{e}_{k}^{c} \\
& =\sum_{j=1}^{n}(-1)^{j-1} a_{j i} \operatorname{det}\left(\phi_{j i}\right) \mathbf{e}_{i}^{c}=(-1)^{i-1} \operatorname{det}(\phi) \mathbf{e}_{i}^{c} .
\end{aligned}
$$

Hence $(\stackrel{n-1}{\wedge} \phi) s \phi^{*}=\operatorname{det}(\phi) s$.

(2) We consider the image of $s^{*} \phi$ in $K_{n-1}(\mathbf{x})^{*}$. Under the isomorphism $R \cong \stackrel{n}{\wedge} R^{n},\left(\mathbf{e}_{i}^{c}\right)^{*}=(-1)^{i-1} \mathbf{e}_{i} \wedge: \stackrel{n-1}{\wedge} R^{n} \rightarrow{ }^{n} \wedge R^{n}$ for $1 \leq i \leq n$. Hence we have $s^{*} \phi\left(\mathbf{e}_{i}\right)=\sum_{j=1}^{n} a_{i j}(-1)^{j-1}\left(\mathbf{e}_{j}^{c}\right)^{*}=\sum_{j=1}^{n} a_{i j} \mathbf{e}_{j} \wedge=\phi\left(\mathbf{e}_{i}\right) \wedge$ as an element of $K_{n-1}(\mathbf{x})^{*}$. Thus we have

$$
\stackrel{n-1}{\wedge} \phi^{*}\left(s^{*} \phi\left(\mathbf{e}_{i}\right)\right)=(\stackrel{n-1}{\wedge} \phi)^{*}\left(s^{*} \phi\left(\mathbf{e}_{i}\right)\right)=\phi\left(\mathbf{e}_{i}\right) \wedge \stackrel{n-1}{\wedge} \phi=(-1)^{i-1} \operatorname{det}(\phi)\left(\mathbf{e}_{i}^{c}\right)^{*} .
$$

Proof of Theorem 4.1. (1) Since grade $((\mathbf{x}))>0, \operatorname{rk}(\phi)<n$ and, thus $\operatorname{det}(\phi)=0$. We consider the following commutative diagram:

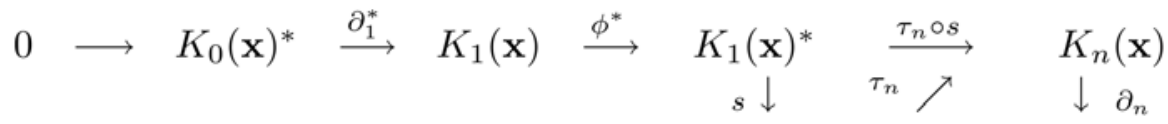

$$
\begin{aligned}
& K_{n-1}(\mathbf{x}) \stackrel{n^{n-1} \phi}{\longrightarrow} K_{n-1}(\mathbf{x}) \text {. }
\end{aligned}
$$


By Lemma 4.2, we have $0=\operatorname{det}(\phi) \cdot s=\wedge^{n-1} \phi \circ s \circ \phi^{*}=\partial_{n} \circ \tau_{n} \circ s \circ \phi^{*}$. On the other hand, by $\operatorname{grade}((\mathbf{x}))>0, \partial_{n}$ is injective and, thus, $\left(\tau_{n} \circ s\right) \circ \phi^{*}=0$. This implies that our sequence

$$
0 \rightarrow K_{0}(\mathbf{x})^{*} \stackrel{\partial_{1}^{*}}{\rightarrow} K_{1}(\mathbf{x})^{*} \stackrel{\phi^{*}}{\rightarrow} K_{1}(\mathbf{x})^{*} \stackrel{\tau_{n} \circ s}{\longrightarrow} K_{n}(\mathbf{x})
$$

is a complex. To prove acyclicity, it is enough to show that $\operatorname{grade}\left(I_{n-1}(\phi)\right) \geq 2$. Then we have that $I_{n-1}(\phi)=I_{1}(\stackrel{n-1}{\wedge} \phi)=I_{1}\left(\partial_{n} \circ \tau_{n}\right)$ $=\left(x_{1}, \cdots, x_{n}\right) I_{1}\left(\tau_{n}\right)$. Since grade $((\mathbf{x})) \geq 3$, we conclude that $\operatorname{grade}\left(I_{n-1}(\phi)\right) \geq 2$ iff $\operatorname{grade}\left(I_{1}\left(\tau_{n}\right)\right) \geq 2$.

(2) By the same construction of (1), we have that $I \cong I_{1}\left(\tau\left(\mathbf{x} ; \phi_{2}^{*}\right)\right)$ and, hence, $I_{n-1}\left(\phi_{2}\right)=\left(x_{1}, \cdots, x_{n}\right) I_{1}\left(\tau\left(\mathbf{x} ; \phi_{2}^{*}\right)\right)$. By our assumption, grade $(I) \geq 2$ and, by the exactness of the free resolution of $I$, $\operatorname{grade}\left(I_{1}\left(\tau\left(\mathbf{x} ; \phi_{2}^{*}\right)\right)\right) \geq 2$. This implies that $I=I_{1}\left(\tau\left(\mathbf{x} ; \phi_{2}^{*}\right)\right)$.

Remark 4.3. As above, if $\mu: K_{i}(\mathbf{x}) \rightarrow K_{i+1}(\mathbf{x})$ such that $\stackrel{i}{\wedge} \phi=\partial_{i+1} \circ \mu$, than $\tau_{j}: K_{j}(\mathbf{x}) \rightarrow K_{j+1}(\mathbf{x})$ such that $\stackrel{j}{\wedge} \phi=\partial_{j+1} \circ \tau_{j}$ for $j>i$ is defined by $\tau_{j}(a \wedge b)=\mu(a) \wedge \stackrel{j-i}{\wedge} \phi(b)$ for $a \in K_{i}(\mathbf{x})$ and $b \in K_{j-i}(\mathbf{x})$. Hence that $I_{1}(\tau(\mathbf{x} ; \phi))$ is determined by the map $\mu$ (and $\mathbf{x}$ ). At this moment, we don't know how to describe it from $\phi$, in general. Accordingly, we study this problem under the most extreme conditions.

Let $\phi: K_{1}(\mathbf{x}) \rightarrow K_{1}(\mathbf{x})$ be an $R$-linear map. We suppose that grade $((\mathbf{x}))>0$ and $\operatorname{Im}(\phi) \subset B_{1}(\mathbf{x})$. Namely, we have an $R$-linear map $Y: K_{1}(\mathbf{x}) \rightarrow K_{2}(\mathbf{x})$ such that $\phi=Y \circ \partial_{2}$ (cf. Theorem 3.1) 


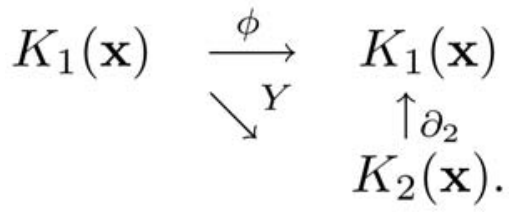

Then we have following commutative diagram:

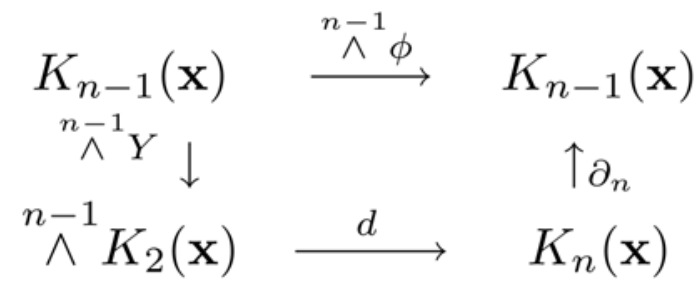

where $d: \stackrel{n-1}{\wedge} K_{2}(\mathbf{x}) \rightarrow K_{n}(\mathbf{x})$ is defined by $d\left(a_{1} \wedge \cdots \wedge a_{n-1}\right)=a_{1} \wedge \partial_{2}\left(a_{2}\right)$ $\wedge \cdots \wedge \partial_{2}\left(a_{n-1}\right)$ for $a_{1}, \cdots, a_{n-1} \in K_{2}(\mathbf{x})$. By the structure of a DG-algebra $\left(K_{\bullet}(\mathbf{x}), \partial_{\bullet}\right)$, it is easy to see that $\partial_{n} \circ d=\wedge^{n-1} \partial_{2}$. Furthermore, since $\partial_{n}$ is injective, $d$ is uniquely determined up to sign under permutations of $a_{1}, \cdots, a_{n-1}$. The element of the image of $d$ is an $n-2$ form of $\mathbf{x}$. Hence that $I(\tau(\mathbf{x} ; \phi))=I_{1}(\stackrel{n-1}{\wedge} Y \cdot d)$ is an ideal generated by $n-2$ forms of $\mathbf{x}$ with coefficients consisting of $n-1$ minors of $Y$.

Now, we give some examples of $I(\tau(\mathbf{x} ; \phi))$ for small number $n$.

Example $4.4(n=3)$. Let $K_{1}\left(x_{1}, x_{2}, x_{3}\right) \rightarrow K_{2}\left(x_{1}, x_{2}, x_{3}\right)$ be a linear map represented by a $3 \times 3$-matrix $Y=\left(y_{i j}\right)$. Consider $\stackrel{2}{\wedge} Y: K_{2}$ $\left(x_{1}, x_{2}, x_{2}\right) \rightarrow \stackrel{2}{\wedge} K_{2}\left(x_{1}, x_{2}, x_{3}\right)$. A basis of $K_{2}\left(x_{1}, x_{2}, x_{2}\right) \quad$ (resp., $\stackrel{2}{\wedge} K_{2}$ $\left.\left(x_{1}, x_{2}, x_{3}\right)\right)$ is ordered by $\mathbf{e}_{12}<\mathbf{e}_{13}<\mathbf{e}_{23}$ (resp., $\mathbf{e}_{12} \wedge \mathbf{e}_{13}<\mathbf{e}_{12} \wedge \mathbf{e}_{23}$ $<\mathbf{e}_{13} \wedge \mathbf{e}_{23}$ ). (Here we denote by $\mathbf{e}_{i j}=\mathbf{e}_{i} \wedge \mathbf{e}_{j} \in K_{2}\left(x_{1}, x_{2}, x_{3}\right)$.) Let $Y_{i j}$ be a $2 \times 2$ matrix obtained by deleting the $i$-th row and the $j$-th column of $Y$. Then we have 


$$
\stackrel{2}{\wedge} Y=\left(\begin{array}{lll}
\left|Y_{33}\right| & \left|Y_{32}\right| & \left|Y_{31}\right| \\
\left|Y_{23}\right| & \left|Y_{22}\right| & \left|Y_{21}\right| \\
\left|Y_{13}\right| & \left|Y_{12}\right| & \left|Y_{11}\right|
\end{array}\right) .
$$

Also, $d: \stackrel{2}{\wedge} K_{2}\left(x_{1}, x_{2}, x_{3}\right) \rightarrow K_{3}\left(x_{1}, x_{2}, x_{3}\right)$ is defined by

$$
\begin{aligned}
& d\left(\mathbf{e}_{12} \wedge \mathbf{e}_{13}\right)=\mathbf{e}_{1} \wedge \mathbf{e}_{2} \wedge\left(x_{1} \mathbf{e}_{3}-x_{3} \mathbf{e}_{1}\right)=x_{1} \mathbf{e}_{1} \wedge \mathbf{e}_{2} \wedge \mathbf{e}_{3}, \\
& d\left(\mathbf{e}_{12} \wedge \mathbf{e}_{23}\right)=\mathbf{e}_{1} \wedge \mathbf{e}_{2} \wedge\left(x_{2} \mathbf{e}_{3}-x_{3} \mathbf{e}_{2}\right)=x_{2} \mathbf{e}_{1} \wedge \mathbf{e}_{2} \wedge \mathbf{e}_{3}, \\
& d\left(\mathbf{e}_{13} \wedge \mathbf{e}_{23}\right)=\mathbf{e}_{1} \wedge \mathbf{e}_{3} \wedge\left(x_{2} \mathbf{e}_{3}-x_{3} \mathbf{e}_{2}\right)=x_{3} \mathbf{e}_{1} \wedge \mathbf{e}_{2} \wedge \mathbf{e}_{3} .
\end{aligned}
$$

Hence we have that

$$
\begin{aligned}
I_{1}(\tau(\mathbf{x} ; \phi)) & =\left(\begin{array}{lll}
Y_{33} \mid & \left|Y_{32}\right| & \left|Y_{31}\right| \\
\left|Y_{23}\right| & \left|Y_{22}\right| & \left|Y_{21}\right| \\
\left|Y_{13}\right| & \left|Y_{12}\right| & \left|Y_{11}\right|
\end{array}\right)\left(\begin{array}{c}
x_{1} \\
x_{2} \\
x_{3}
\end{array}\right) \\
& =\left(\begin{array}{ccc}
y_{11} & y_{12} & y_{13} \\
y_{21} & y_{22} & y_{23} \\
x_{3} & -x_{2} & x_{1}
\end{array}|,| \begin{array}{ccc}
y_{11} & y_{12} & y_{13} \\
x_{3} & -x_{2} & x_{1} \\
y_{31} & y_{32} & y_{33}
\end{array}|,| \begin{array}{lll}
x_{3} & -x_{2} & x_{1} \\
y_{21} & y_{22} & y_{23} \\
y_{31} & y_{32} & y_{33}
\end{array} \mid\right) .
\end{aligned}
$$

Example 4.5 [Proof of Theorem $1.1(n=4)]$.

Let $K_{1}\left(x_{1}, x_{2}, x_{3}, x_{4}\right) \rightarrow K_{2}\left(x_{1}, x_{2}, x_{3}, x_{4}\right)$ be a linear map represented by a $4 \times 6$-matrix $Y=\left(y_{i j}\right)$. We regard that indices of columns of $Y$ correspond to $\mathbf{e}_{12}<\mathbf{e}_{13}<\mathbf{e}_{14}<\mathbf{e}_{23}<\mathbf{e}_{24}<\mathbf{e}_{34}$, where $\mathbf{e}_{i j}=\mathbf{e}_{i} \wedge \mathbf{e}_{j}$. In the same way as $n=3$, we compute images of $d: \stackrel{3}{\wedge} K_{2}(\mathbf{x}) \rightarrow K_{4}(\mathbf{x})$ for each $\mathbf{e}_{i j} \wedge \mathbf{e}_{k l} \wedge \mathbf{e}_{p q}$. Then, a coefficient of $\mathbf{e}_{1} \wedge \mathbf{e}_{2} \wedge \mathbf{e}_{3} \wedge \mathbf{e}_{4}$ corresponds to $\mathbf{e}_{i j} \wedge \mathbf{e}_{k l} \wedge \mathbf{e}_{p q}(i j \cdot k l \cdot p q$ in short) is listed as follows: 


\begin{tabular}{|c|c|c|c|}
\hline $\begin{array}{c}12 \cdot 13 \cdot 14 \\
x_{1}^{2}\end{array}$ & $\begin{array}{c}12 \cdot 13 \cdot 24 \\
x_{1} x_{2}\end{array}$ & $\begin{array}{c}12 \cdot 13 \cdot 34 \\
x_{1} x_{3}\end{array}$ & $\begin{array}{c}12 \cdot 14 \cdot 34 \\
x_{1} x_{4}\end{array}$ \\
\hline $12 \cdot 14 \cdot 23$ & $12 \cdot 23 \cdot 24$ & $12 \cdot 23 \cdot 34$ & $12 \cdot 24 \cdot 34$ \\
$-x_{1} x_{2}$ & $x_{2}^{2}$ & $x_{2} x_{3}$ & $x_{2} x_{4}$ \\
\hline $13 \cdot 14 \cdot 23$ & $13 \cdot 23 \cdot 24$ & $13 \cdot 23 \cdot 34$ & $13 \cdot 24 \cdot 34$ \\
$-x_{1} x_{3}$ & $x_{2} x_{3}$ & $x_{3}^{2}$ & $x_{3} x_{4}$ \\
\hline $13 \cdot 14 \cdot 24$ & $14 \cdot 23 \cdot 24$ & $14 \cdot 23 \cdot 34$ & $14 \cdot 24 \cdot 34$ \\
$-x_{1} x_{4}$ & $x_{2} x_{4}$ & $x_{3} x_{4}$ & $x_{4}^{2}$ \\
\hline
\end{tabular}

and $\quad d\left(\mathbf{e}_{i j} \wedge \mathbf{e}_{k l} \wedge \mathbf{e}_{p q}\right)=0 \quad$ for $\quad i j \cdot k l \cdot p q=12 \cdot 13 \cdot 23,12 \cdot 14 \cdot 24$, $13 \cdot 14 \cdot 34,23 \cdot 24 \cdot 34$. Consider the correspondence of indices of columns of $Y$, we have that $d \circ \wedge Y\left(\mathbf{e}_{i} \wedge \mathbf{e}_{j} \wedge \mathbf{e}_{k}\right)=\mathbf{x} \cdot\left(\begin{array}{cccc}{[i j k ; 123]} & {[i j k ; 125]} & {[i j k ; 126]} & {[i j k ; 136]} \\ -[i j k ; 134] & {[i j k ; 145]} & {[i j k ; 146]} & {[i j k ; 156]} \\ -[i j k ; 234] & {[i j k ; 245]} & {[i j k ; 246]} & {[i j k ; 256]} \\ -[i j k ; 235] & {[i j k ; 345]} & {[i j k ; 346]} & {[i j k ; 356]}\end{array}\right) \cdot \mathbf{x}^{t}$, for all $1 \leq i<j<k \leq 4$ and the image of $d \circ \stackrel{3}{\wedge} Y$ coincides with $I(\mathbf{x} ; Y)$ of Theorem 1.1.

Next, we describe relations of $R(I(\mathbf{x} ; Y))$. Denote by $\mathbf{y}_{i}$ the $i$-th column of $Y$ for $1 \leq i \leq 6$ and $\mathbf{t}=\left(\begin{array}{llll}T_{1} & T_{2} & T_{3} & T_{4}\end{array}\right)$. Since the first syzygy of $I(\mathbf{x} ; Y)$ is given by $\partial_{2}^{*} \circ Y^{t}$, if we put $\mathbf{f}:=\left(f_{1} f_{2} f_{3} f_{4}\right)=\mathbf{t} Y \cdot \partial_{2}$, then

$$
\mathbf{f}^{t}=\left(\begin{array}{cccc}
0 & -\mathbf{t y}_{1} & -\mathbf{t y}_{2} & -\mathbf{t y}_{3} \\
\mathbf{t y}_{1} & 0 & -\mathbf{t y}_{4} & -\mathbf{t y}_{5} \\
\mathbf{t y}_{2} & \mathbf{t y}_{4} & 0 & -\mathbf{t y}_{6} \\
\mathbf{t y}_{3} & \mathbf{t y}_{5} & \mathbf{t y}_{6} & 0
\end{array}\right)\left(\begin{array}{l}
x_{1} \\
x_{2} \\
x_{3} \\
x_{4}
\end{array}\right)
$$


By Lemma 2.2, if $I(\mathbf{x} ; Y)$ is generically a complete intersection of $\operatorname{ht}(I(\mathbf{x} ; Y))=2, r\left(I(\mathbf{x} ; Y)_{\mathfrak{p}}\right) \leq 1$ for $\mathfrak{p} \in V(I(\mathbf{x} ; Y))$ of $\operatorname{ht}(\mathfrak{p})=3$, and grade $\left(I_{1}(\mathbf{x})\right) \geq 3$, then the defining ideal of $R(I(\mathbf{x} ; Y))$ is given by $\operatorname{Pf}(M)$, where

$$
M=\left(\begin{array}{c|cccc}
0 & x_{1} & x_{2} & x_{3} & x_{4} \\
\hline-x_{1} & 0 & \mathbf{t y}_{6} & -\mathbf{t y}_{5} & \mathbf{t y}_{4} \\
-x_{2} & -\mathbf{t y}_{6} & 0 & \mathbf{t y}_{3} & -\mathbf{t y}_{2} \\
-x_{3} & \mathbf{t y}_{5} & -\mathbf{t y}_{2} & 0 & \mathbf{t y}_{1} \\
-x_{4} & -\mathbf{t y}_{4} & \mathbf{t y}_{3} & -\mathbf{t y}_{1} & 0
\end{array}\right) .
$$

For all $1 \leq i, j \leq 4$, we set $Y^{(i, j)}=\left(\begin{array}{cccc}0 & y_{i 6} & -y_{i 5} & y_{i 4} \\ -y_{i 6} & 0 & y_{j 3} & -y_{j 2} \\ y_{i 5} & -y_{j 3} & 0 & y_{j 1} \\ -y_{i 4} & y_{j 2} & -y_{j 1} & 0\end{array}\right)$.

Since $\mathrm{pf}_{1}(M)=\mathbf{t}\left(\mathbf{y}_{6} \mathbf{y}_{1}^{t}+\mathbf{y}_{5} \mathbf{y}_{2}^{t}+\mathbf{y}_{4} \mathbf{y}_{3}^{t}\right) \mathbf{t}^{t}$, the content ideal of $\mathrm{pf}_{1}(M)$ is given by $c\left(\operatorname{pf}_{1}(M)\right)=\left(\operatorname{pf}\left(\left\{Y^{(i, i)}\right)\right\}_{1 \leq i \leq 4},\left\{\operatorname{pf}\left(Y^{(i, j)}\right)+\operatorname{pf}\left(Y^{(j, i)}\right)\right\}_{1 \leq i<j \leq 4}\right)$.

Finally, we arrange the statement of Theorem 1.1, (1), as follows.

Suppose that $I(\mathbf{x} ; Y)$ satisfies the following conditions:

(1) $I(\mathbf{x} ; Y)$ is generically a complete intersection of $\mathrm{ht}(I(\mathbf{x} ; Y))=2$.

(2) $\operatorname{ht}((\mathbf{x})) \geq 3$.

(3) $r\left(I(\mathbf{x} ; Y)_{\mathfrak{p}}\right)=0$ for $\mathfrak{p} \in V(I(\mathbf{x} ; Y)) \backslash V(\mathbf{x})$ of $\operatorname{ht}(\mathfrak{p})=3$.

(4) $\operatorname{ht}\left((\mathbf{x})+\left(\operatorname{pf}\left(\left\{Y^{(i, i)}\right)\right\}_{1 \leq i \leq 4},\left\{\operatorname{pf}\left(Y^{(i, j)}\right)+\operatorname{pf}\left(Y^{(j, i)}\right)\right\}_{1 \leq i<j \leq 4}\right)\right) \geq 4$.

Then $R(I(\mathbf{x} ; Y))$ is a Gorenstein ring of $\operatorname{pd}_{S}(R(I))=3$ (cf. Remark 3.3). 
To describe generators of $I_{1}(\tau(\mathbf{x} ; \phi))$, we put some notations. We denote a complete graph with $n$ vertices $V=\left\{v_{1}, \cdots, v_{n}\right\}$ by $K_{n}$ and we fix an order of edges $E\left(K_{n}\right)$ of $K_{n}$. For a spanning tree $T$ of $K_{n}$, if we denote edges of $T$ in order of $E\left(K_{n}\right)$ by $\left\{v_{i_{1}} v_{j_{1}}<\cdots<v_{i_{n-1}} v_{j_{n-1}} \mid i_{p}<j_{p}\right.$, $p=1, \cdots, n-1\}$, then we have an equation $\mathbf{e}_{i_{1}} \wedge \mathbf{e}_{j_{1}} \wedge\left(\mathbf{e}_{j_{2}}-\mathbf{e}_{i_{2}} \wedge\right.$ $\cdots \wedge\left(\mathbf{e}_{j_{n-1}}-\mathbf{e}_{i_{n-1}}\right)=(-1)^{s} \mathbf{e}_{1} \wedge \mathbf{e}_{2} \wedge \cdots \wedge \mathbf{e}_{n}$ for some $s>0$. We define a sign of $T$ by $\operatorname{sgn}(T)=(-1)^{s}$. Also, we denote a monomial of $\mathbf{x}$ in degree $2^{\#} E(T)-n=n-2$ by $x^{T}=\prod_{i=1}^{n} x_{i}^{\operatorname{deg}_{T}\left(v_{i}\right)-1}$.

For an $n \times \frac{n(n-1)}{2} \quad R$-matrix $Y$, we regard indices of columns correspond to $E\left(K_{n}\right)$ and denote $n \times(n-1)$ matrix consisting columns correspond to $E(T)$ by $Y^{T}$. Also, denote by $Y_{i}$ a matrix obtained by deleting the $i$-th row of $Y$. Then $I_{1}(\tau(\mathbf{x} ; \phi))$ is described as follows.

Theorem 4.6. Let $T C$ be a set of all spanning tree of $K_{n}$. If $\operatorname{Im}(\phi) \subset B_{1}(\mathbf{x})$, then

$$
I_{1}(\tau(\mathbf{x} ; \phi))=\left(\sum_{T \in T C} \operatorname{sgn}(T) \operatorname{det}\left(Y_{i}^{T}\right) x^{T} \mid 1 \leq i \leq n\right) .
$$

Proof. First note that if $v_{i_{1}} v_{i_{2}}, v_{i_{2}} v_{i_{3}}, \cdots, v_{i_{p}} v_{i_{1}}\left(i_{1}<i_{2}<\cdots<i_{p}\right)$ is a cycle in $K_{n}$, then $\mathbf{e}_{i_{1}} \wedge \mathbf{e}_{i_{2}} \wedge\left(\mathbf{e}_{i_{3}}-\mathbf{e}_{i_{2}}\right) \wedge \cdots \wedge\left(\mathbf{e}_{i_{p}}-\mathbf{e}_{i_{1}}\right)=0$. Suppose that $d\left(\mathbf{e}_{i_{1} j_{1}} \wedge \cdots \wedge \mathbf{e}_{i_{n-1} j_{n-1}}\right) \neq 0$. Then $\left\{v_{i_{1}} v_{j_{1}}, \cdots, v_{i_{n-1}} v_{j_{n-1}}\right\}$ has no cycle and, since $\left\{i_{1}, j_{1}, \cdots, i_{n-1}, j_{n-1}\right\}=\{1, \cdots, n\}$, there exists a spanning tree $T$ of $K_{n}$ such that $E(T)=\left\{v_{i_{1}} v_{j_{1}}, \cdots, v_{i_{n-1}} v_{j_{n-1}}\right\}$. Then it is enough to show that a coefficient of $d\left(\mathbf{e}_{T}\right)$ is $\pm x^{T}$, where $\mathbf{e}_{T}=\wedge_{k=1}^{n-1} \mathbf{e}_{i_{k} j_{k}}$. Since $T$ is a tree, $T$ has a terminal vertex $v_{i_{k}}$ (or $v_{j_{k}}$ ), i.e., $\operatorname{deg}_{T}\left(v_{i_{k}}\right)=1$ (or $\operatorname{deg}_{T}\left(v_{j_{k}}\right)=1$ ). Note that $d$ is uniquely determined up to sign under permutations of $\left\{\mathbf{e}_{i_{p} j_{p}}\right\}$. Thus we have 


$$
\begin{gathered}
d\left(\mathbf{e}_{T}\right)= \pm d\left(\mathbf{e}_{T \backslash\left\{v_{i k}\right\}}\right) \wedge \partial_{2}\left(\mathbf{e}_{i_{k} j_{k}}\right)= \pm d\left(\mathbf{e}_{T \backslash\left\{v_{i k}\right\}}\right) \wedge x_{j_{k}} \mathbf{e}_{i_{k}} \\
\left(\text { or } \pm d\left(\mathbf{e}_{T \backslash\left\{v_{j_{k}}\right\}}\right) \wedge x_{i_{k}} \mathbf{e}_{j_{k}}\right) \text {. Hence, by induction on } n \text {, we have } \\
d\left(\mathbf{e}_{T \backslash\left\{v_{i_{k}}\right\}}\right)= \pm x^{T \backslash\left\{v_{i_{k}}\right\}} \wedge{ }_{i \neq i_{k}} \mathbf{e}_{i}\left(\text { or } d\left(\mathbf{e}_{T \backslash\left\{v_{j_{k}}\right\}}\right)= \pm x^{T \backslash\left\{v_{j_{k}}\right\}} \wedge{ }_{i \neq j_{k}} \mathbf{e}_{i}\right) .
\end{gathered}
$$

This complete the proof.

\section{Examples}

Example 5.1. Let $f: A=k[x, y, z, w, v] \rightarrow k\left[t_{1}, t_{2}, t_{3}\right]$ such that $f(x)=t_{1}^{6}, f(y)=t_{2}^{6}, f(z)=t_{3}^{6}, f(w)=t_{1}^{4} t_{2} t_{3}, f(v)=t_{1}^{2} t_{2}^{3} t_{3}$ and put $I=\operatorname{Ker}(f)$. Then $I$ is generically a complete intersection of $\operatorname{ht}(I)=2$ and $A$ / I has a free resolution $0 \rightarrow A \stackrel{\phi_{3}}{\rightarrow} A^{4} \stackrel{\phi_{2}}{\rightarrow} A^{4} \stackrel{\phi_{1}}{\rightarrow} A$, where

$$
\phi_{1}=\left(\begin{array}{c}
y w^{3}-x v^{3} \\
x^{4} y z-w^{6} \\
x^{3} y^{2} z-w^{3} v^{3} \\
x^{2} y^{3} z-v^{6}
\end{array}\right), \quad \phi_{2}=\left(\begin{array}{cccc}
x^{2} y^{2} z & 0 & v^{3} & -w^{3} \\
-x^{3} y z & -v^{3} & w^{3} & 0 \\
w^{3} & y & -x & 0 \\
-v^{3} & 0 & -y & x
\end{array}\right),
$$

and $\phi_{3}=\left(\begin{array}{llll}x & y & v^{3} & w^{3}\end{array}\right)$. Then $\phi_{2}^{*}$ is decomposed by $\phi_{2}^{*}=Y \cdot \partial_{2}$

$$
=\left(\begin{array}{cccccc}
-x^{2} y z & 0 & 0 & 0 & 0 & -1 \\
0 & 0 & 0 & 1 & 0 & 0 \\
0 & -1 & 0 & 0 & -1 & 0 \\
0 & 0 & 1 & 0 & 0 & 0
\end{array}\right)\left(\begin{array}{cccc}
-y & x & 0 & 0 \\
-v^{3} & 0 & x & 0 \\
-w^{3} & 0 & 0 & x \\
0 & -v^{3} & y & 0 \\
0 & -w^{3} & 0 & y \\
0 & 0 & -w^{3} & v^{3}
\end{array}\right) .
$$


Thus we have $\operatorname{Im}\left(\phi_{2}^{*}\right) \subset B_{1}\left(x, y, v^{3}, w^{3}\right)$. By the form of $\phi_{2}$, it is easy to see that $\mu\left(I_{\mathfrak{p}}\right) \leq 3$ for all $\mathfrak{p} \in V(I)$ such that $\mathfrak{p} \not\left(x, y, v^{3}, w^{3}\right)$ and, also, ht $\left(x, y, v^{3}, w^{3}\right)=4$. Thus $R(I)$ is a Gorenstein (cf. Example 4.5).

Linear equations $x^{2} y^{2} z T_{1}+v^{3} T_{3}-w^{3} T_{4},-x^{3} y z T_{1}+y T_{2}-x T_{3}$, $w^{3} T_{1}+y T_{2}-x T_{3},-v^{3} T_{1}-y T_{3}+x T_{4}$ of $R(I)$ are expressed an element of $B_{1}\left(x, y, w^{3}, v^{3} ; S\right)$ as

$$
\left(\begin{array}{cccc}
0 & x^{2} y z T_{1} & T_{3} & -T_{4} \\
-x^{2} z y T_{1} & 0 & -T_{2} & T_{3} \\
-T_{3} & T_{2} & 0 & T_{1} \\
T_{4} & -T_{3} & -T_{1} & 0
\end{array}\right)\left(\begin{array}{c}
x \\
y \\
v^{3} \\
w^{3}
\end{array}\right) .
$$

Hence we have that

$\operatorname{Pf}\left(\begin{array}{c|cccc}0 & x & y & v^{3} & w^{3} \\ \hline-x & 0 & -T_{1} & T_{3} & T_{2} \\ -y & T_{1} & 0 & T_{4} & T_{3} \\ -v^{3} & -T_{3} & -T_{4} & 0 & -x^{2} y z T_{1} \\ -w^{3} & -T_{2} & -T_{3} & x^{2} y z T_{1} & 0\end{array}\right)$

defines $R(I)$.

Example 5.2. Let $A=k[x, y, x]$ and put $I=\left(x^{a}, x^{b}, y^{d}, y^{c}, y^{d} z\right)$ with positive integers $a>b$ and $c>d$. Then $A / I$ has a free resolution $0 \rightarrow A \stackrel{\phi_{3}}{\rightarrow} A^{4} \stackrel{\phi_{2}}{\rightarrow} A^{4} \stackrel{\phi_{1}}{\rightarrow} A$, where 


$$
\phi_{1}=\left(\begin{array}{c}
x^{a} \\
x^{b} y^{d} \\
y^{c} \\
y^{d} z
\end{array}\right), \phi_{2}=\left(\begin{array}{cccc}
y^{d} & -x^{a-b} & 0 & 0 \\
0 & y^{c-d} & -x^{b} & 0 \\
0 & z & 0 & -x^{b} \\
0 & 0 & z & -y^{c-d}
\end{array}\right), \phi_{3}=\left(\begin{array}{llll}
0 & z & y^{c-d} & x^{b}
\end{array}\right) .
$$

Since $(-z, 0,0,0),\left(-y^{c-d}, 0,0,0\right),\left(-x^{b}, 0,0,0\right) \in B_{1}\left(0, z, y^{c-d}, x^{b} ; S\right)$, $\operatorname{Im}\left(\phi_{2}^{*}\right)$ is contained in $B_{1}\left(0, z, y^{c-d}, x^{b} ; A\right)$ if and only if $a \geq 2 b$ and $2 d \geq c$. Assume that $a \geq 2 b$ and $2 d \geq c$. Then we have

$$
\left(\begin{array}{c}
y^{d} T_{1}-x^{a-b} T_{2} \\
y^{c-d} T_{2}-x^{b} T_{3} \\
z T_{2}-x^{b} T_{4} \\
z T_{3}-y^{c-d} T_{4}
\end{array}\right)=\left(\begin{array}{cccc}
0 & 0 & y^{2 d-c} T_{1} & -x^{a-2 b} T_{2} \\
0 & 0 & -T_{2} & -T_{3} \\
-y^{2 d-c} T_{1} & T_{2} & 0 & -T_{4} \\
-x^{a-2 b} T_{2} & T_{3} & T_{4} & 0
\end{array}\right)\left(\begin{array}{c}
0 \\
z \\
y^{c-d} \\
x^{b}
\end{array}\right) .
$$

Put

$$
M=\operatorname{Pf}\left(\begin{array}{c|cccc}
0 & 0 & z & y^{c-d} & x^{b} \\
\hline 0 & 0 & T_{4} & -T_{3} & T_{2} \\
-z & -T_{4} & 0 & -x^{a-2 b} T_{2} & y^{2 d-c} T_{1} \\
-y^{c-d} & T_{3} & x^{a-2 b} T_{2} & 0 & 0 \\
-x^{b} & -T_{2} & -y^{2 d-c} T_{1} & 0 & 0
\end{array}\right) .
$$

Note that $r(I) \neq 0$, since $\ell(I) \leq \operatorname{dim}(A)=3$. Hence, by Example 4.5, $R(I)$ is a Gorenstein if and only if $c\left(\operatorname{pf}_{1}(M)\right)=A$, i.e., either $a=2 b$ or $c=2 d$.

In the following, we construct an ideal $I$ with $\mu(I)=n$ such that $R(I)$ is Gorenstein for every integer $n \geq 4$. Let $(A, \mathfrak{m})$ be a Gorenstein local ring and let $J=\left(a_{1}, \cdots, a_{n}\right)$ be an ideal of $A$. Let $a \in A$ be a super 
regular element for $J$ and $b=\sum_{i=1}^{n} c_{i} a_{i} \in \mathfrak{m} J$ such that $a, b$ forms a regular sequence. We define $I=(a J, b)=J \cap(a, b)$ and $\varphi: S=A$ $\left[T_{1}, \cdots, T_{n}, T\right] \rightarrow R(I)$ by $\varphi\left(T_{i}\right)=a a_{i} t$ for $1 \leq i \leq n$ and $\varphi(T)=b t$.

Proposition 5.3. $R(I) \cong R(J)[T] /\left(a T-\sum_{i=1}^{n} c_{i} T_{i}\right)$

Proof. If $F \in \operatorname{Ker}(\varphi)$ of $\operatorname{deg}(F)=l$, then it can be expressed as $F=T F_{1}-F_{2}$ such that $F_{1} \in S_{l-1}$ and $F_{2} \in A\left[T_{1}, \cdots, T_{n}\right]_{l}$. Now, we prove $F \in\left(\operatorname{Ker}(\varphi) \cap A\left[T_{1}, \cdots, T_{n}\right]\right)+\left(a T-\sum_{i=1}^{n} c_{i} T_{i}\right)=: \mathfrak{a}$ by induction on $l$.

Since $b \varphi\left(F_{1}\right) t=\varphi\left(F_{2}\right) \in a^{l} J^{l} t^{l}$ and $a, b$ is a regular sequence, we have that $\varphi\left(F_{1}\right) t^{-l+1} \in\left(a^{l}\right) \cap I^{l-1}$ and $\left(a^{l}\right) \cap I^{l-1}=a^{l} J^{l-1}$ by induction on $l$. Indeed, we have following equations; $\left(a^{l}\right) \cap I^{l-1}=\left(a^{l}\right) \cap\left(a^{l-1} J^{l-1}\right.$ $\left.+b I^{l-2}\right)=\left(a^{l}\right) \cap\left(a^{l-1} J^{l-1}+\left(a^{l-1}\right) \cap b I^{l-2}\right)=\left(a^{l}\right) \cap\left(a^{l-1} J^{l-1}+b\left(\left(a^{l-1}\right) \cap\right.\right.$ $\left.\left.I^{l-2}\right)\right)=\left(a^{l}\right) \cap\left(a^{l-1} J^{l-1}+b a^{l-1} J^{l-2}\right)=\left(a^{l}\right) \cap a^{l-1} J^{l-1}=a^{l} J^{l-1}$, since $a$ is a super regular and $a, b$ is a regular sequence. Hence $\varphi\left(F_{1}\right)=a^{l} z t^{l-1}$ for some $z \in J^{l-1}$ and there exist $G \in A\left[T_{1}, \cdots, T_{n}\right]_{l-1}$ such that $\varphi\left(F_{1}\right)=a \varphi(G)$. By induction hypothesis, $F_{1}-a G \in \mathfrak{a}$ and, hence, $F=T F_{1}-F_{2}=T\left(F_{1}-a G\right)+G\left(a T-\sum_{i=1}^{n} c_{i} T_{i}\right)+G \sum_{i=1}^{n} c_{i} T_{i}-F_{2} \in \mathfrak{a}$.

Corollary 5.4. $R(I)$ is Cohen-Macaulay (resp., Gorenstein) if and only if so is $R(J)$.

Proposition 5.5. Suppose that $J$ is generically a complete intersection ideal of $\mathrm{ht}(J)=2$. Then $I$ is also generically a complete intersection of $\mathrm{ht}(I)=2$ satisfying following conditions: 
(1) $\operatorname{depth}(A / I)=\operatorname{depth}(A / J)$ and $\mu(I)=\mu(J)+1$.

(2) $\ell(I)=\ell(J)+1$ and $r(I)=r(J)$.

(3) $b_{i}(I)=b_{i}(J)+1(i=0,1)$ or $b_{i}(J)$ (otherwise).

Proof. First, we show that $I$ is generically a complete intersection. Let $\mathfrak{p} \supset I$ be a minimal prime of $I$. If $a \notin \mathfrak{p}$, then $I_{\mathfrak{p}}=J_{\mathfrak{p}}$ and $\mu\left(I_{\mathfrak{p}}\right)=2$. Suppose that $a \in \mathfrak{p}$ and so that $\mathfrak{p}$ is a minimal prime of $(a, b)$. If $J \subset \mathfrak{p}$, then $(a, J) \subset \mathfrak{p}$ and $h t(\mathfrak{p}) \geq 3$, since a is a super regular on $J$. This contradicts the minimality of $\mathfrak{p}$. Hence $J \not \subset \mathfrak{p}$ and $I_{\mathfrak{p}}=(a, b)_{\mathfrak{p}}$.

(1) Since $J=[I: a]$ and $\mathfrak{m} J=[\mathfrak{m} I: a]$, we have exact sequences $0 \rightarrow A / J \stackrel{a}{\rightarrow} A / I \rightarrow A /(a, b) \rightarrow 0$ and $0 \rightarrow J / \mathfrak{m} J \stackrel{a}{\rightarrow} I / \mathfrak{m} I \rightarrow(b) /$ $\mathfrak{m}(b) \rightarrow 0$. It follows that $\operatorname{depth}(A / I)=\operatorname{depth}(A / J)$ and $\mu(I)=\mu(J)+1$. (Note that $\operatorname{dim}(A) \geq 3$, since $a$ is a super regular on $J$.)

(2) The assertion follows from $R(I) / \mathfrak{m} R(I)=(R(J) / \mathfrak{m} R(J))[T]$.

(3) Let $\mathbb{F}_{\bullet}$ be a minimal graded $S$-free resolution of $R(J)[T]$ and $F=a T-\sum_{i=1}^{n} c_{i} T_{i}$. Then a mapping cone $C(F)=\operatorname{cone}\left(\mathbb{F}_{\bullet}(-1) \stackrel{F}{\rightarrow} \mathbb{F}_{\bullet}\right)$ gives a minimal graded $S$-free resolution of $R(I)$. Hence $[C(F)]_{1}=\left[\mathbb{F}_{\bullet}\right]_{1}$ $\oplus\left[\left(\mathbb{F}_{\bullet}(-1)\right)[-1]\right]_{1}=\left[\mathbb{F}_{\bullet}\right]_{1} \oplus A[-1]$ is a minimal $A$-free resolution of $I$.

Example 5.6. Combing Theorem 2.1 and Proposition 5.5, if $J$ is a perfect ideal of $\operatorname{ht}(J)=2$ such that $J$ is generated by a $d$-sequence, then $I$ (in Proposition 5.3) is also generated by a $d$-sequence. In the following, we give a typical example of it.

Let $I=\left(x^{\alpha_{1}}, \cdots, x^{\alpha_{m}}\right) \subset A=k\left[x_{1}, \cdots, x_{d}\right]$ be a perfect monomial ideal of $\operatorname{ht}(I)=2$. We show that $I$ is generated by a $d$-sequence, if $I$ is square-free. 
Let $\xi: \oplus_{i=1}^{m} A \mathbf{e}_{i} \rightarrow A$ be an $A$-linear map defined by $\xi\left(\mathbf{e}_{i}\right)=x^{\alpha_{i}}$ for $1 \leq i \leq m$. Then $\operatorname{Ker}(\xi)$ is generated by $m-1$ elements of the form

$$
x^{\beta_{1}} \mathbf{e}_{i_{1}}-x^{\beta_{1}^{\prime}} \mathbf{e}_{j_{1}}, \cdots, x^{\beta m-1} \mathbf{e}_{i_{m-1}}-x^{\beta_{1}^{\prime}} \mathbf{e}_{j_{m-1}},
$$

where $x^{\alpha_{i_{p}}+\beta_{p}}=x^{\alpha_{j_{p}}+\beta_{p}^{\prime}}=\operatorname{lcm}\left(x^{\alpha_{i_{p}}}, x^{\alpha_{j_{p}}}\right)$ for $1 \leq p \leq m-1$. We define a graph $\mathcal{G}$ on $\left\{\mathbf{e}_{1}, \cdots, \mathbf{e}_{m}\right\}$ with edges $\left\{\mathbf{e}_{i_{1}} \mathbf{e}_{j_{1}}, \cdots, \mathbf{e}_{i_{m-1}} \mathbf{e}_{j_{m-1}}\right\}$.

For any $i, j$, if we put $x^{\delta}=\operatorname{lcm}\left(x^{\alpha_{i}}, x^{\alpha_{j}}\right)$, then $x^{\delta-\alpha_{j}} \mathbf{e}_{i}-x^{\delta-\alpha_{i}} \mathbf{e}_{j}$ is expressed as an $A$-linear combination of $m-1$ generators as above. This implies that $\mathcal{G}$ is connected and, thus, it is a tree. Therefore, $\mathcal{G}$ has a terminal vertex $\mathbf{e}_{1}$ (after renumbering vertices). Then we may assume that $I$ is represented by the matrix of the form

$$
\left(\begin{array}{c|cc}
x^{\beta_{1}} & -x^{\beta_{1}^{\prime}} & 0 \cdots 0 \\
\hline 0 & & \\
\vdots & & N \\
0 & &
\end{array}\right),
$$

where $N$ is an $m-2 \times m-1 A$-matrix and $\operatorname{gcd}\left(x^{\beta_{1}}, x^{\beta_{1}^{\prime}}\right)=1$.

Assume that $I=\left(x^{\beta_{1}^{\prime}} \operatorname{det}\left(N_{1}\right), x^{\beta_{1}} I_{m-2}(N)\right)$ is square free. $\left(N_{1}\right.$ is an $m-2 \times m-2$ matrix obtained by deleting the first column of $N$.) Then $I_{m-2}(N)$ is also square free and $x^{\beta_{1}}$ is a super regular element on $I_{m-1}(N)$, since $x^{\beta_{1}}$ has no common indeterminates with generators of $I_{m-2}(N)$. Also $x^{\beta_{1}}, x^{\beta_{1}^{\prime}} \operatorname{det}\left(N_{1}\right)$ forms a regular sequence, since $\operatorname{gcd}\left(x^{\beta_{1}}, x^{\beta_{1}^{\prime}}\right)=1$. Hence, by Proposition 5.3 and induction on $m, R(I)$ is CI. 


\section{References}

[1] L. Avramov, Complete intersections and symmetric algebras, J. Alg. 73 (1981), 248-263.

[2] D. Buchsbaum and D. Eisenbud, What makes complex exact?, J. Algebra 25 (1973), 84-139.

[3] D. Buchsbaum and D. Eisenbud, Algebra structures for finite free resolutions and some structure theorems for ideals of codimension 3, Amer. J. Math. 99 (1977), 447-485.

[4] S. Goto and S. Iai, Gorenstein graded rings associated to ideals, J. Algebra 294(2) (2005), 373-407.

[5] S. Goto and Y. Nakamura, On the Gorensteinness in graded rings associated to ideals of analytic deviation one, Contemp. Math. 159 (1994), 51-72.

[6] S. Goto and Y. Shimoda, On the Gorensteinness of Rees and form rings of almost complete intersections, Nagoya Math. J. 92 (1983), 69-88.

[7] S. Hukaba and C. Huneke, Rees algebras of ideals having small analytic deviation, Trans. Amer. Math. Soc. 339 (1993), 373-402.

[8] C. Huneke, On the symmetric and Rees of ideal Generated by $d$-sequences, J. Alg. 62 (1980), 268-275.

[9] C. Huneke, The Koszul homology of an ideal, Adv. Math. 56 (1985), 295-318.

[10] A. Kustin, The minimal free resolutions of the Huneke-Ulrich deviation two Gorenstein ideals, Proc. AMS J. Alg. 100 (1986), 265-304.

[11] S. Morey, Equations of blow up ideals of codimension two and three, J. Pure and Appl. Alg. 109 (1996), 197-211.

[12] S. Morey and B. Ulrich, Rees algebras of ideals with low codimension, Proc. AMS 124(12) (1996), 3653-3661.

[13] A. Simis, B. Ulrich and W. Vasconcelos, Cohen-Macaulay Rees algebras and degrees of polynomial relations, Math. Ann. 301 (1995).

[14] B. Ulrich and W. Vasconcelos, The equations of Rees algebras of ideals with linear presentation, Math. Z. 214 (1993), 79-92.

[15] W. Vasconcelos, Arithmetic of blowup algebras, London Math. LNS 195 (1994). 